\title{
The protective effects of the miR-129-5p/keap-1/Nrf2 axis on Ang II-induced cardiomyocyte hypertrophy
}

\author{
Huiming Ye, Guiyu Xu, Dexian Zhang, Rupeng Wang \\ Department of Cardiology, Beijing Shijitan Hospital, Capital Medical University, Beijing, China \\ Contributions: (I) Conception and design: H Ye; (II) Administrative support: H Ye; (III) Provision of study materials or patients: G Xu; (IV) Collection \\ and assembly of data: H Ye, G Xu; (V) Data analysis and interpretation: D Zhang, R Wang; (VI) Manuscript writing: All authors; (VII) Final approval \\ of manuscript: All authors. \\ Correspondence to: Huiming Ye. Department of Cardiology, Beijing Shijitan Hospital, Capital Medical University, No. 10 Tieyi Road, Haidian \\ District, Beijing 100038, China. Email: miuhey@126.com.
}

\begin{abstract}
Background: Cardiac hypertrophy is a common pathological process in many cardiac diseases, and persistent cardiac hypertrophy is the main cause of heart failure and sudden cardiogenic death. Thus, it is essential to elucidate the mechanism of cardiac hypertrophy to ensure better prevention and treatment.

Methods: The Human cardiac myocytes (HCMs) were incubated with $100 \mathrm{nmol} / \mathrm{L}$ Ang II (Sigma) for 48 hours to induce the in vitro cardiomyocyte hypertrophy model. The $[(3 \mathrm{H}])$-leucine incorporation assay was used to evaluate cardiomyocytes hypertrophy. The activities of oxidative stress related enzymes superoxide dismutase (SOD), catalase (CAT), malondialdehyde (MDA) and nitric oxide (NO) were detected using corresponding detection kits following standard protocol. Targeting relationship was verified through Bioinformatics analysis and luciferase reporter gene assay. The morphological change of cardiomyocyte was observed through immunofluorescence staining. Expressions of message ribonucleic acid (mRNA) and proteins were detected by quantitative real-time polymerase chain reaction and western blot, respectively.

Results: In our study, the suppressed expression of micro ribonucleic acid (miRNA)-129-5p and the elevated expression of kelch-like ECH-associated protein 1 (keap-1) were found in the angiotensin II (Ang II) -induced cardiomyocyte hypertrophy model. MiR-129-5p effectively mimics suppressed Ang II-induced hypertrophic responses and oxidative stress. The results also showed that keap-1 was a target of miR-129-5p, and that the miR-129-5p inhibitor promoted cardiomyocyte hypertrophy and oxidative stress by elevating keap-1. Additionally, small interfering RNA (siRNA)-keap-1 activated the nuclear factor erythroid2-related factor 2 (Nrf2) pathway, while the miR-129-5p inhibitor inactivated the Nrf2 pathway by further elevating keap-1. The addition of the Nrf2 pathway activator NK-252 largely weakened the promoting effects of the miR-129-5p inhibitor on the progression of cardiomyocyte hypertrophy by suppressing oxidative stress.

Conclusions: In general, the results indicate that the overexpression of miRNA-129-5p protects against cardiomyocyte hypertrophy by targeting keap-1 via the Nrf2 pathway.
\end{abstract}

Keywords: Cardio hypertrophy; oxidative stress; miR-129a-5p; keap-1; Nrf2 pathway

Submitted Nov 25, 2020. Accepted for publication Jan 21, 2021.

doi: $10.21037 / \mathrm{atm}-20-8079$

View this article at: http://dx.doi.org/10.21037/atm-20-8079

\section{Introduction}

Cardiac hypertrophy is a common pathological process in many cardiac diseases, such as long-term hypertension and hypertrophic cardiomyopathy. Early cardiac hypertrophy is accompanied by the compensatory enlargement of cardiomyocyte size and the thickening of the ventricular walls to maintain cardiac function under the stimulation of various physiological and pathological factors. However, 
persistent cardiac hypertrophy could further induce heart failure and sudden cardiogenic death $(1,2)$. To date, the potential molecular mechanism of cardiac hypertrophy has been poorly understood, which has greatly hindered associated clinic treatments. Thus, it is essential that the mechanism of cardiac hypertrophy be elucidated to enable better prevention and treatment.

Previous research has indicated that angiotensin II (Ang II) is the most important active hormone in the Renin-Angiotensin-Aldosterone System (RAAS) and the strongest inducer of cardiac hypertrophy (3). By binding to Angiotensin II type I receptors, Ang II activates many of the signaling molecules involved in cardiac hypertrophy (4). The continuous activation of RAAS can induce cardiac hypertrophy, fibrosis, necrosis, and the oxidative stress response, and further lead to cardiac function gradually transforming from compensation to de-compensation (5). Thus, Ang II sustained-release stimulation-induced cardiac hypertrophy is an ideal research model.

Gene therapy is an effective and safe treatment method that can compensate for defective genes or correct abnormal genes (6). With the development of molecular biology, molecular genetics, immunology, and other related disciplines, the use of gene therapy to treat a variety of diseases, including cardiovascular diseases, has developed rapidly $(7,8)$. In recent years, many micro ribonucleic acids (miRNAs) have been considered targets of gene therapy in many diseases $(9,10)$. It has been confirmed that ectopic expressed miRNAs are commonly found in the cardiac hypertrophy model and defective human heart samples (11). For example, the decreased expression of both micro ribonucleic acid (miRNA)-133 and miR-1 was found in mouse and human models of cardiac hypertrophy (12). Additionally, Li et al. demonstrated that overexpression of miR-199a inhibited autophagy in cardiomyocyte and further induced cardiac hypertrophy in vivo (13). However, the mechanisms by which miR-129-5p affects the progression of cardiac hypertrophy have not been thoroughly studied.

Nuclear factor erythroid2-related factor 2 (Nrf2) is an important transcription factor that regulates antioxidant stress and plays a cardiac protection role (14). Chen et al. found that Nrf2 deficiency aggravated Ang II-induced cardiac injury by increasing hypertrophy and enhancing inflammation (15). The Kelch-like ECH-associated protein 1 (Keap1), an intracellular sensor for oxidative stress, is a specific receptor for $\mathrm{Nrf} 2$ that mediates the degradation of Nrf2 through ubiquitination (16). The dissociation of the Keap1/Nrf2 complex promotes the nuclear transfer of
Nrf2, thus inducing the activation of the Nrf2 pathway (17). Luteolin was demonstrated to attenuate cardiac ischemia/ reperfusion injury in diabetic rats by antioxidant via the Keap1/Nrf2 signaling (18). Thus, the keap-1-Nrf2 signaling pathway might also play an important role in cardiac hypertrophy by regulating oxidative stress.

In the present study, we found that the overexpression of miR-129-5p alleviated Ang II-induced cardiomyocyte hypertrophy and oxidative stress in a cardiomyocyte hypertrophy model in vitro. A further study revealed that the miR-129-5p mimic exerted a protective effect by targeting keap-1 by activating the Nrf2 pathway. This is the first study to identify the mechanisms of miR-129-5p in cardiac hypertrophy. These results provide novel targets for further research and the treatment of cardiac hypertrophy.

We present the following article in accordance with the MDAR reporting checklist (available at http://dx.doi. org/10.21037/atm-20-8079).

\section{Methods}

\section{Statement}

The present study was approved by the Ethics Committee of the Beijing Shijitan Hospital of Capital Medical University [Approval NO. SYXK (Beijing) 2017-0025].

All the operations in this study are in accordance with the operation standards and regulations formulated by Beijing Shijitan Hospital of Capital Medical University.

\section{Cell culture and reagents}

Human cardiac myocytes (HCMs) were purchased from ScienCell, United States of America (USA; Cat. \#6200, original from normal myocardial tissue of aborted fetus, cell purity higher than $90 \%$ without human immunodeficiency virus-1, Hepatitis B Virus, Hepatitis C Virus, mycoplasma, bacteria, yeast and fungi) and were maintained in a humidified incubator at $37{ }^{\circ} \mathrm{C} / 5 \%$ carbon dioxide. Nrf2 and keap-1 antibodies were purchased from Santa Cruz (Shanghai, China. Nrf2 Cat.: sc-365949; keap-1 Cat.: sc365626). NK-252 (Sigma-Aldrich, Saint Louis, MO, USA) was used to activate the Nrf2 pathway.

\section{Angiotensin II-induced cardiomyocyte bypertrophy in vitro}

In our experiments, the HCMs were incubated with $100 \mathrm{nmol} / \mathrm{L}$ Ang II (Sigma-Aldrich, Saint Louis, MO, 
USA) for 48 hours to induce the in vitro cardiomyocyte hypertrophy model. The serum-free medium containing Ang II was changed every 24 hours.

\section{Cell transfection}

MiR-129-5p mimic and miR-129-5p NC were purchased from GenePharma (Shanghai, China). SiRNA-miR-129-5p (5'-GCAAGCCCAGACCGCAAAAAG-3'), siRNAkeap-1 and corresponding siRNA-NC were designed and synthesized by Invitrogen. HCMs were transfected with miR-129-5 p mimics or miR-129-5p mimics nonspecific control (NC) for 72 hours for the experiments in accordance with the instructions of Lipofectamine 2000 (Invitrogen, Carlsbad, CA, USA).

\section{Quantitative real-time polymerase chain reaction}

The total ribonucleic acid (RNA) in HCMs was isolated using the TRIzol reagent (Invitrogen) in accordance with the manufacturer's instructions. Reverse transcription was conducted with the reverse transcriptase amplification kit (Fermentas, New York, USA). Specific reverse transcription primers and quantitative PCR primers were obtained from RiboBio Co., Ltd. (Guangzhou, China). A quantitative realtime polymerase chain reaction (qRT-PCR) was performed by the SYBR Premix DimerEraser on a 7900HT system. Rnu6 (U6) and glyceraldehyde phosphate dehydrogenase (GAPDH) were regarded as the internal references for miRNAs and messenger RNAs (mRNAs), respectively. The qRT-PCR results were analyzed by the $2^{-\Delta \Delta C t}$ method (19). The following primers were used: MiR-129-5p (forward, 5 '-ACACTCCTTTTTGCGTCTGGGCTTGC-3' and reverse, 5'-TGGTGTCGTGGAGTCG-3'), Keap1 (forward, 5'-AGTTACTTGTCCCGGTCCTG-3' and reverse, 5'-TTTCCAACTCCGCACAAAGG-3'), Nppa (forward, 5'-ATGGGCTCCTTCTCCATCAC-3' and reverse, 5'-TCTTCGGTACCGGAAGCTG-3') and Myh7 (forward, 5'-GCAGCTTATCAGGAAGGAATAC-3' and reverse, 5'-CTTGCGTACTCTGTCACTC-3').

\section{Western blot}

The protein in the HCMs was extracted using an icecold radio-Immunoprecipitation Assay (RIPA) buffer (Beyotime, Shanghai, China). Protein concentration was valued using the bicinchoninic acid (BCA) protein assay kit (Cwbiotech, China). The protein sample was mixed with loading buffer and incubated for 8 minutes in a boiling water bath, the average protein $(40 \mu \mathrm{g})$ was then split by $10 \%$ sodium dodecyl sulphate (SDS) polyacrylamide minigel, and then transferred onto the Polyvinylidene Fluoride (PVDF) membranes (Millipore, Massachusetts, USA), and blocked with $5 \%$ skim milk for 1 hour at room temperature. The PVDF membranes were then incubated with the primary antibodies and the corresponding horseradish peroxidase (HRP)-conjugated secondary antibodies. Finally, the membranes were treated with an enhanced chemiluminescence detection kit (Super Signal West Pico; Pierce), and the proteins were analyzed using the image processing program ImageJ. Glyceraldehyde 3-phosphate dehydrogenase (GAPDH) was used as an endogenous reference.

\section{(3H)-leucine incorporation assay}

The $(3 \mathrm{H})$-leucine incorporation assay was used to evaluate cardiomyocytes hypertrophy [as previously described (20)]. Briefly, the HCMs were seeded in 96-well plates at the density of $5 \times 10^{4}$ cells/well and were transfected with miR129-5p mimics or miR-129-5p NC as indicated. Next, $0.1 \mu \mathrm{M}$ Ang II was used to stimulate cardiomyocyte hypertrophy and $1 \mu \mathrm{Ci}(3 \mathrm{H})$-leucine was added to each well. After stimulation with Ang II for 60 hours, the cells were harvested by precipitation with $10 \%$ trichloroacetic acid on ice for 30 minutes, and were then solubilized with $1 \mathrm{~mol} / \mathrm{L}$ $\mathrm{NaOH}$ overnight at $4{ }^{\circ} \mathrm{C}$. The samples were neutralized with $1 \mathrm{~mol} / \mathrm{L} \mathrm{HCl}$, and the level of $(3 \mathrm{H})$ was determined by using a $\beta$ counter to assess ( $3 \mathrm{H})$-leucine incorporation.

\section{Detection of superoxide dismutase, catalase, malondialdehyde, nitric oxide levels}

The activities of oxidative stress-related enzymes superoxide dismutase (SOD), catalase (CAT), malondialdehyde (MDA), and nitric oxide (NO) were detected using corresponding detection kits in accordance with the manufacturers' instructions. The kits of SOD, CAT, and MDA were purchased from Nanjing Jiancheng Bioengineering Institute. The NO kit was purchased from Beyotime Biotechnology.

For the detection of SOD: the samples were mixed with WST-1 working solution and enzyme working solution for $20 \mathrm{~min}$ at $37^{\circ} \mathrm{C}$. At last, SOD activity was analyzed with microplate reader at $450 \mathrm{~nm}$.

For the detection of CAT, the samples were incubated 
with working solution at $25^{\circ} \mathrm{C}$ for $5 \mathrm{~min}$. Then, hydrogen peroxide $\left(\mathrm{H}_{2} \mathrm{O}_{2}\right)$ solution was added into the wells for $30 \mathrm{~min}$ at $25^{\circ} \mathrm{C}$. The stop solution and developer mix were added in turn and were incubate for $10 \mathrm{~min}$ at $25^{\circ} \mathrm{C}$. At last, CAT activity was analyzed with microplate reader at $570 \mathrm{~nm}$.

For the detection of MDA: TBA solution was mixed with the samples at $95^{\circ} \mathrm{C}$ for $60 \mathrm{~min}$. Then, the mixture was kept in ice bath for $10 \mathrm{~min}$. Then, the mixture was transferred to the wells of microplate and was analyzed with microplate reader at $532 \mathrm{~nm}$. For the detection of NO: the samples were mixed with nitrate reductase and enzyme cofactor for 1 hour at room temperature to convert nitrate into nitrite. Enhancer was then added into the mixture for $10 \mathrm{~min}$ at room temperature, followed by Griess Reagent R1 and Griess Reagent R2. At last, NO was also analyzed with microplate reader at $540 \mathrm{~nm}$.

\section{Measurement of reactive oxygen species (ROS) production}

The production of ROS was detected using the fluorescent probe dichloro-dihydro-fluorescein diacetate (DCFHDA) (Sigma, USA). After treated as indicated, HCMs were incubated with $50 \mu \mathrm{M}$ DCFH-DA at $37{ }^{\circ} \mathrm{C}$ for $30 \mathrm{~min}$ avoid the light. Then, the cells were washed twice using cold PBS. The fluorescence of ROS was detected by the microplate reader [ $\operatorname{Ex}(\lambda) 485 \mathrm{~nm}$; $\operatorname{Em}(\lambda) 535 \mathrm{~nm}$ ] and average fluorescence intensity was analyzed using the Image $\mathrm{J}$ analysis system (National Institutes of Health).

\section{Bioinformatics analysis}

The potential targets of miR-129-5p were predicted by performing a search of the following online database: miRanda (http://www.microrna.org/microrna/home.do), TargetScan (http://www.targetscan.org/) and miRmap (http://mirmap.ezlab.org/app/).

\section{Luciferase reporter gene assay}

The HCMs were first inoculated into 12-well plates at a density of $1 \times 10^{5}$ cells per well. The 3 - untranslated region (3'-UTR) of the Keap-1 gene containing putative miR129-5p targeting site was amplified by chemical synthesis and was inserted into the psiCHECK2 vector (Promega, Madison, WI, USA). When the confluence reached $70 \%$, the HCMs were transfected with related mixtures, including 50 ng keap-1 wild-type or keap-1 mutant-type 3'-UTR reporter plasmids, miR-129-5p mimics or miR-129-5p $\mathrm{NC}$ in a final concentration of $20 \mathrm{nM}$, and Lipofectamine 2000 for 48 hours. Luciferase activity was detected using the dual-luciferase reporter gene kit (Beyotime, Shanghai, China).

\section{Immunofluorescence}

For the immunofluorescence staining, the HCMs were incubated with the primary antibody against $\alpha$-actinin (1:200, Sigma, St. Louis, Missouri, USA) and the fluorescent secondary antibody (1:200, Alexa Fluor 488, Invitrogen) in accordance with the instructions. Nuclear staining was conducted with Hoechst (Sigma, St. Louis, Missouri, USA). The cells were observed by a fluorescence microscope (Zeiss, Heidenheim, Baden-Wuerttemberg, Germany).

\section{Statistical analysis}

A statistical analysis was conducted using SPSS 21.0 (SPSS Inc., IL, USA). A Student's $t$-test was used to compare the two independent groups. Differences among multiple groups were carried out by a one - way analysis of variance followed by a Student-Newman-Keuls post-hoc test. P values of $<0.05$ were considered statistically significant.

\section{Results}

\section{The overexpression of miR-129-5p inhibits Angiotensin II-induced cardiomyocyte bypertrophy}

Ang II-treated HCMs were transfected with the miR-129$5 \mathrm{p}$ mimic or siRNA-NC. The relative expression of miR129-5p and keap-1 mRNA was detected through qRTPCRs. The results showed that the expression of miR129-5p was inhibited by Ang II, and was then elevated by the presence of the miR-129-5p mimic (see Figure 1A). Conversely, the expression of keap- 1 was induced by Ang II stimulation and was suppressed by miR-129-5p overexpression detected through both qRT-PCR (see Figure 1B) and western blot (see Figure 1C). Additionally, immunofluorescence staining showed that the cell surface area was obviously increased by Ang II stimulation and decreased by the miR-129-5p mimic (see Figure 1D). The expression change of myocardial hypertrophy marker genes Nppa and Myh7 also demonstrated that Ang II-induced cardiomyocyte hypertrophy was obviously relieved by the miR-129-5p mimic (see Figure 1E). Further, Ang II-induced 
A
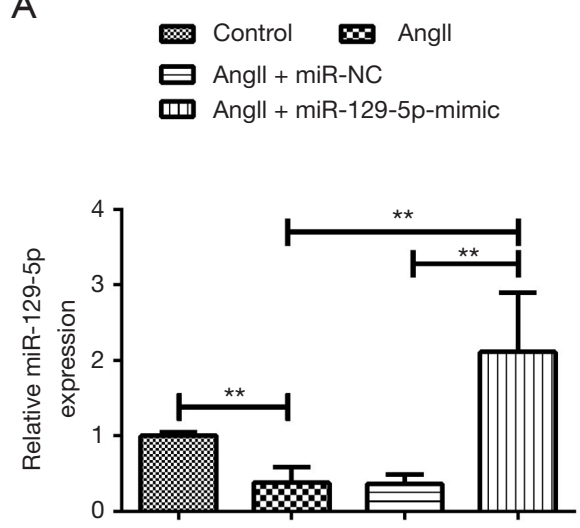

C
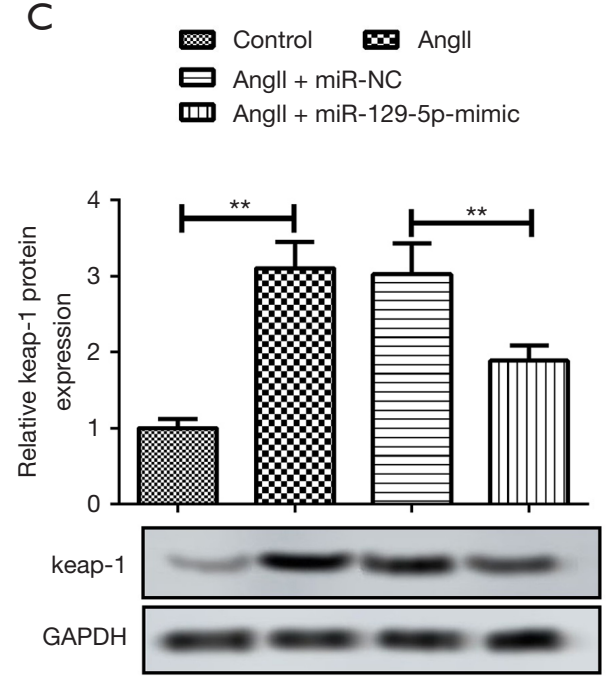

$\mathrm{E}$
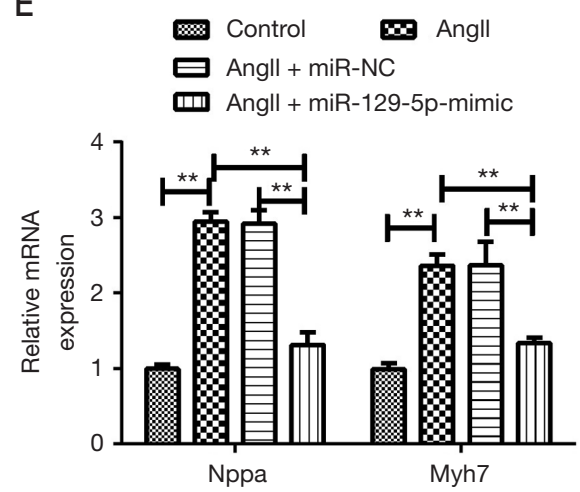

B
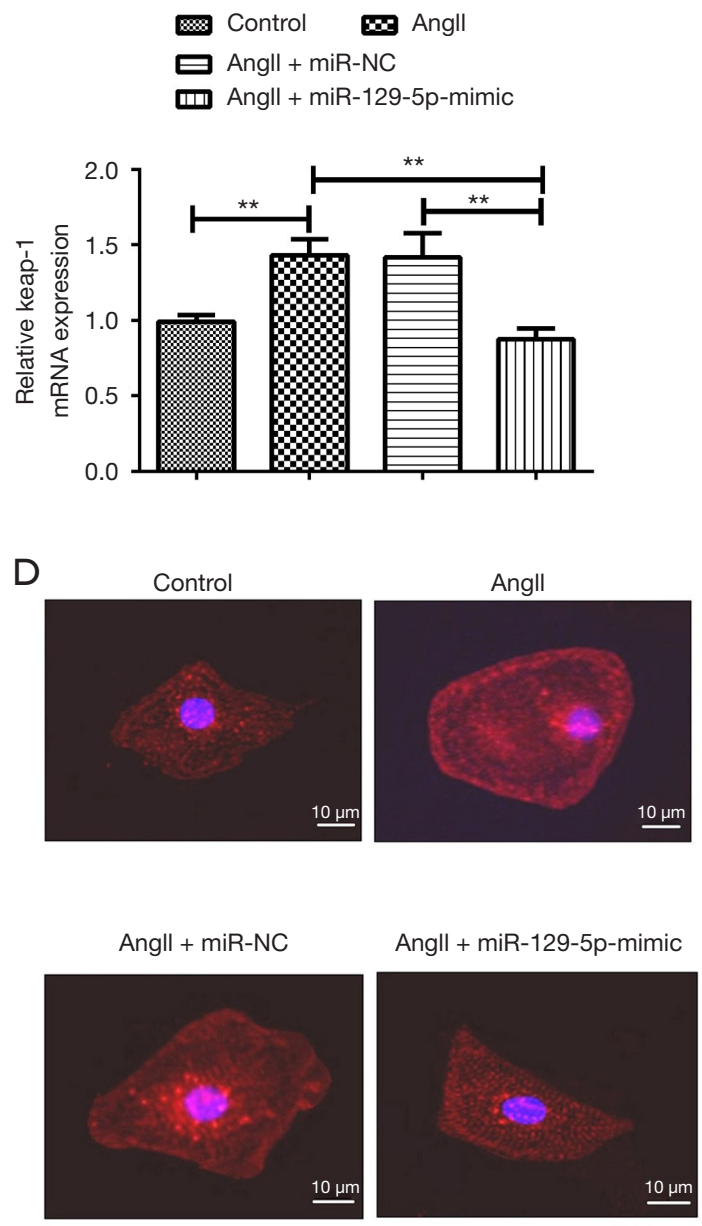

Angll + miR-129-5p-mimic

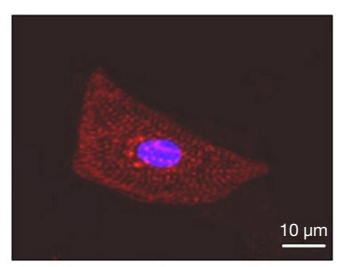

$\mathrm{F}$
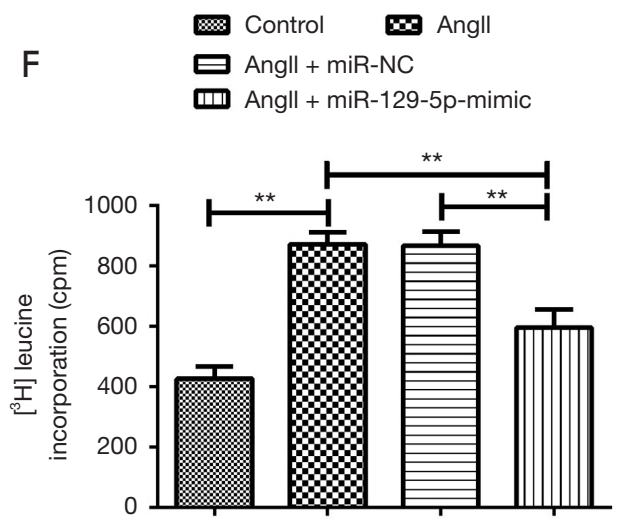

Figure 1 The overexpression of miR-129-5p inhibits Ang II-induced cardiomyocyte hypertrophy. The expression of miR-129-5p (A) was detected through qRT-PCR. The expression of keap-1 mRNA was detected through qRT-PCR (B) and western blot (C), respectively. Immunofluorescence staining showed the morphological changes of cardiomyocytes in different groups (D). Scale is $10 \mu \mathrm{m}$. The expression of myocardial hypertrophy marker genes Nppa and Myh7 was detected through qRT-PCR (E). The (3H)-leucine incorporation assay was conducted to value cardiomyocyte hypertrophy $(\mathrm{F}) .{ }^{* *}, \mathrm{P}<0.01$. The bars show the mean \pm standard deviation (SD) for each of the three independent experiments. miR, micro-RNA; NC, negative control; Ang II, angiotensin II; mRNA, message ribonucleic acid. 
A

$$
\begin{aligned}
& \text { Control Angll } \\
& \text { 므 Angll + miR-NC } \\
& \text { m Angll + miR-129-5p-mimic }
\end{aligned}
$$

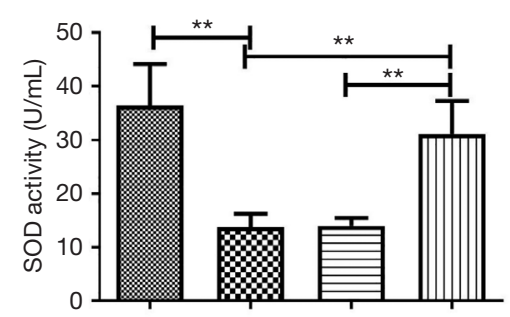

D
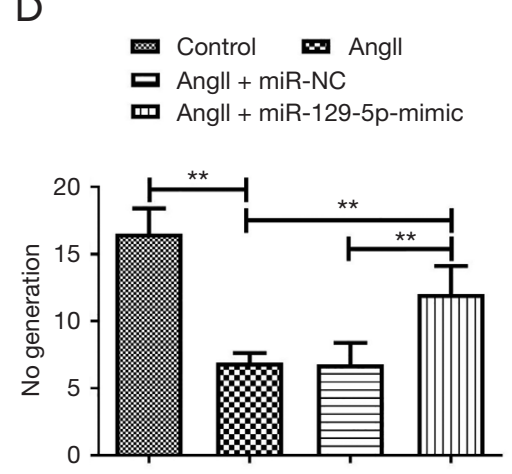

B
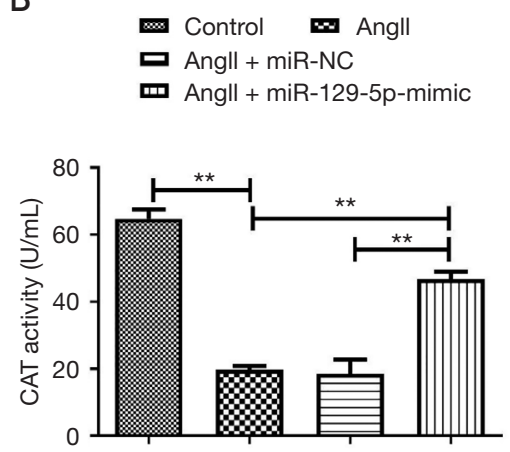

E

$$
\begin{aligned}
& \text { Control } \rightarrow \text { Angll } \\
& \text { Angll }+ \text { miR-NC } \\
& \text { ㄷngll + miR-129-5p-mimic }
\end{aligned}
$$

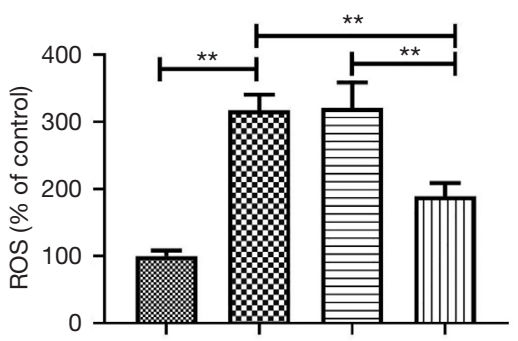

C
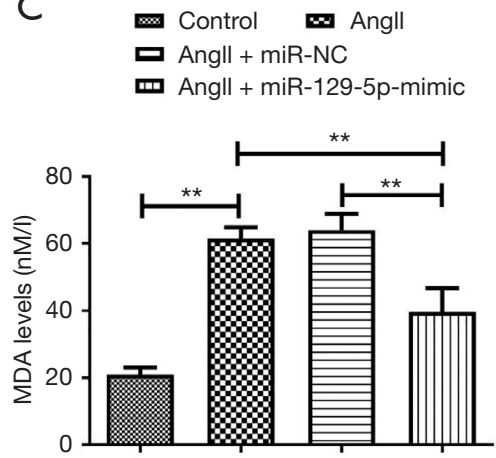

Figure 2 The overexpression of miR-129-5p suppresses Ang II-induced oxidative stress. The activities of oxidative stress-related enzymes SOD (A), CAT (B), and MDA (C), NO (D) and the production of ROS (E) were detected using the corresponding detection kits in accordance with the manufacturer's instructions. ${ }^{* *}, \mathrm{P}<0.01$. The bars show the mean \pm standard deviation (SD) for each of the three independent experiments. miR, micro-RNA; NC, negative control; Ang II, angiotensin II; SOD, superoxide dismutase; CAT, catalase; MDA, malondialdehyde; NO, nitric oxide; ROS, reactive oxygen species.

(3H)-leucine incorporation was significantly decreased after the treatment by the miR-129-5p mimic, which also indicated that miR-129-5p overexpression inhibited cardiomyocyte hypertrophy (see Figure $1 F$ ). The above results demonstrate that the overexpression of miR-129-5p inhibits Ang II-induced cardiomyocyte hypertrophy.

\section{The overexpression of miR-129-5p suppresses Angiotensin} II-induced oxidative stress

Ang II-induced oxidative stress is another important inducer of cardiomyocyte hypertrophy. The results showed that the activities of the antioxidant enzymes SOD and CAT were strongly destroyed by Ang II stimulation and were then partly recovered by the miR-129-5p mimic (see Figure $2 A, B)$. Additionally, the accumulation of MDA was increased by Ang II and was cleared by the miR-129-5p mimic (see Figure 2C). Conversely, the generation of NO was suppressed by Ang II and was increased by the miR129-5p mimic (see Figure 2D). More directly, detection about ROS showed that the production of ROS was induced by Ang II and was suppressed by the miR-129-5p mimic (see Figure 2E). The above results all indicate that the overexpression of miR-129-5p effectively suppresses Ang II-induced oxidative stress.

\section{Kelch-like ECH-associated protein is a target of miR-129-5p}

As stated above, miRNA exerts a regulating effect by specifically binding to the 3'UTR of mRNA. Thus, the potential targets of miR-129-5p were first predicted through 
A

\begin{tabular}{|c|c|c|}
\hline keap-1-Wt & $5^{\prime}$ & uuuuguuucuuggGCAAAAAu \\
\hline miR-129-5p & $3^{\prime}$ & cguucgggucuggCGUUUUUc \\
\hline keap-1-Mut & $5^{\prime}$ & uuuuguuucuuggCGUUUUUc \\
\hline
\end{tabular}

C

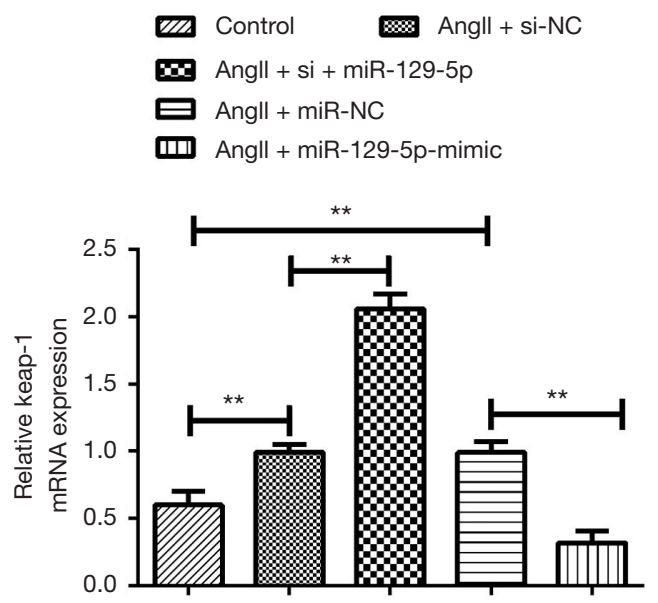

B

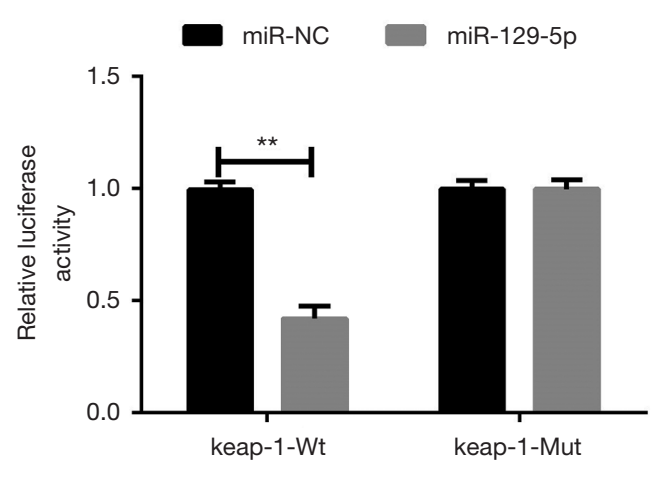

D $\quad 20$ Angll + si-NC

Ex Angll + si + miR-129-5p

E Angll + miR-NC

메 Angll + miR-129-5p-mimic

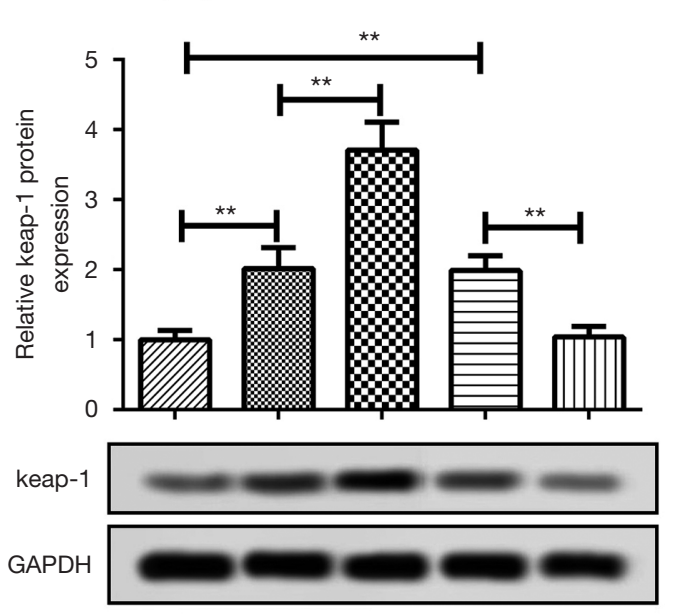

Figure 3 Keap-1 is a target of miR-129-5p. A bioinformatics analysis showed predictive complementary sequences between keap-1 and miR129-5p (A). A luciferase reporter gene assay was conducted to further demonstrate the targeting relationship between keap-1 and miR-129$5 \mathrm{p}$ (B). qRT-PCR (C) and western blot (D) were employed to detect the expression of keap-1. GAPDH was used as an internal reference. **, $\mathrm{P}<0.01$. The bars show the mean \pm standard deviation (SD) for each of the three independent experiments. WT, wild type; MUT, mutant; miR, micro-RNA; keap-1, kelch-like ECH-associated protein 1; NC, negative control; Ang II, angiotensin II; si, small interfering RNA.

a bioinformatics analysis. Figure $3 \mathrm{~A}$ shows the predictive complementary sequences. Further, as the luciferase reporter gene assay in Figure $3 B$ shows, the intensity of luminescence had a more obvious decrease in keap-1wild type $(W T)+$ miR-129-5p mimic group than miR129-5p NC. However, the combination of keap-1- mutant (MUT) and both the miR-129-5p mimic and miR-129-5p
NC had no effect on the intensity of luminescence. Thus, a targeting relationship between keap-1 and miR-129-5p appears to exist. In relation to qRT-PCR (see Figure 3C) and the western blot (see Figure 3D), the results showed that the expression of keap- 1 could be elevated by siRNAmiR-129-5p and suppressed by the miR-129-5p mimic; thus, keap-1 acts as a target of miR-129-5p. 
The MiR-129-5p inhibitor promotes cardiomyocyte hypertrophy and oxidative stress by elevating the Kelchlike ECH-associated protein

We further explored the effects of the miR-129-5p/keap1 axis in cardio hypertrophy. As Figure $4 A$ shows, the elevated keap-1 expression in Ang II group was decreased by siRNA-keap-1, but was largely elevated again by the presence of siRNA-miR-129-5p. Further, the results of immunofluorescence staining shows that siRNA-keap-1 decreased the cell surface area of cardiomyocyte (see Figure 4B), which was previously increased by Ang II. The co-transfection of siRNA-miR-129-5p enlarged the cell surface area of cardiomyocyte compared to that of the Ang II + siRNA-keap-1 group. Thus, the miR-129-5p inhibitor was found to promote cardiomyocyte hypertrophy by elevating keap-1. Additionally, the detection for the expressions of Nppa and Myh7, and the (3H)-leucine incorporation assay further indicated cardiomyocyte hypertrophy in different groups (see Figure $4 C$ and D, respectively). The above results demonstrate that the miR129-5p inhibitor promotes cardiomyocyte hypertrophy by elevating keap-1.

Similarly, the activities of oxidative stress-related enzymes SOD (see Figure 4E) and CAT (Figure 4F) were weaker in the Ang II + siRNA-keap-1 + siRNA-miR-129-5p group than the Ang II + siRNA-keap-1 group. Additionally, the expression of MDA (Figure $4 G$ ) was elevated and the production of NO (Figure $4 H$ ) was decreased in the siRNAmiR-129-5p group compared to that of the Ang II + siRNAkeap-1 group. In the same way, the production of ROS was induced by Ang II and was suppressed by siRNA-keap-1. Subsequently, suppressed production of ROS was elevated in the presence of siRNA-miR-129-5p (see Figure 4I). Thus, the miR-129-5p inhibitor appears to promote oxidative stress by elevating keap-1.

\section{The Kelch-like ECH-associated protein mediated the inactivation of the nuclear factor erythroid 2-related factor pathway by the miR-129-5p inbibitor}

As keap-1 is known to be a specific receptor for $\mathrm{Nrf2}$, and the Nrf2 pathway is closely associated with oxidative stress, we also explored whether the miR-129-5p inhibitor affects cardiomyocyte hypertrophy via the Nrf2 pathway. The results of the western blot showed that the expression of nuclear Nrf2 was suppressed by Ang II, but elevated by the addition of siRNA-keap-1 (see Figure 5A). Additionally, the elevated expression of nuclear Nrf2 was once again suppressed by siRNA-miR-129-5p. Thus, it appears that silenced miR-129-5p could inactivate the Nrf2 pathway by elevating keap-1.

To further verify our ratiocination, the Nrf2 activator NK-252 was employed in a number of experiments. As Figure $5 B$ shows, compared with the Ang II + si-miR-129-5p group, the Nrf2 pathway in Ang II + si-miR-129-5p + NK-252 group was obviously activated by NK-252, which promoted the nuclear transport of Nrf2. Similarly, NK-252 further enhanced the activation of the Nrf2 pathway, which showed higher activation in the Ang II+si-keap-1 group than the Ang II+NC group. The results in relation to the detection of Nppa and Myh7 (see Figure 5C) and the $(3 \mathrm{H})$-leucine incorporation assay (see Figure 5D) both showed that cardiomyocyte hypertrophy was aggravated by $\mathrm{si}-\mathrm{miR}-129-5 \mathrm{p}$ and was relieved in the presence of sikeap-1. Additionally, the protective effects of si-keap-1 were enhanced by NK-252. Further, oxidative stress-related detection showed that the activities of SOD (see Figure 5E) and CAT (see Figure $5 F$ ) were suppressed by si-miR-129-5p, but were then partly recovered by si-keap-1 and NK-252 . Additionally, the accumulated MDA was cleared by NK-252 (see Figure $5 G$ ), and the generation of $\mathrm{NO}$ was increased by si-keap-1 and NK-252 (see Figure 5H). These above results showed that silenced $\mathrm{miR}-129-5 \mathrm{p}$ aggravated cardiomyocyte hypertrophy by promoting keap-1 mediated oxidative stress via the inactivation of $\mathrm{Nrf2}$ pathway. Thus, the overexpression of miR-129-5p appears to alleviate Ang II-induced cardiomyocyte hypertrophy by activating the Nrf2 pathway.

\section{Discussion}

In recent years, there has been an increasing incidence of cardiovascular diseases, which represents a serious threat to human health. Cardiac hypertrophy is a compensatory response of the heart whereby the thickness of the walls increases under the stimulation of various pathological factors (21). Early cardiac hypertrophy is conducive to the maintenance of the normal function of the heart; however, sustained cardiac hypertrophy will eventually lead to heart failure and sudden death (22). Thus, research on the mechanism of cardiac hypertrophy is of great significance, 


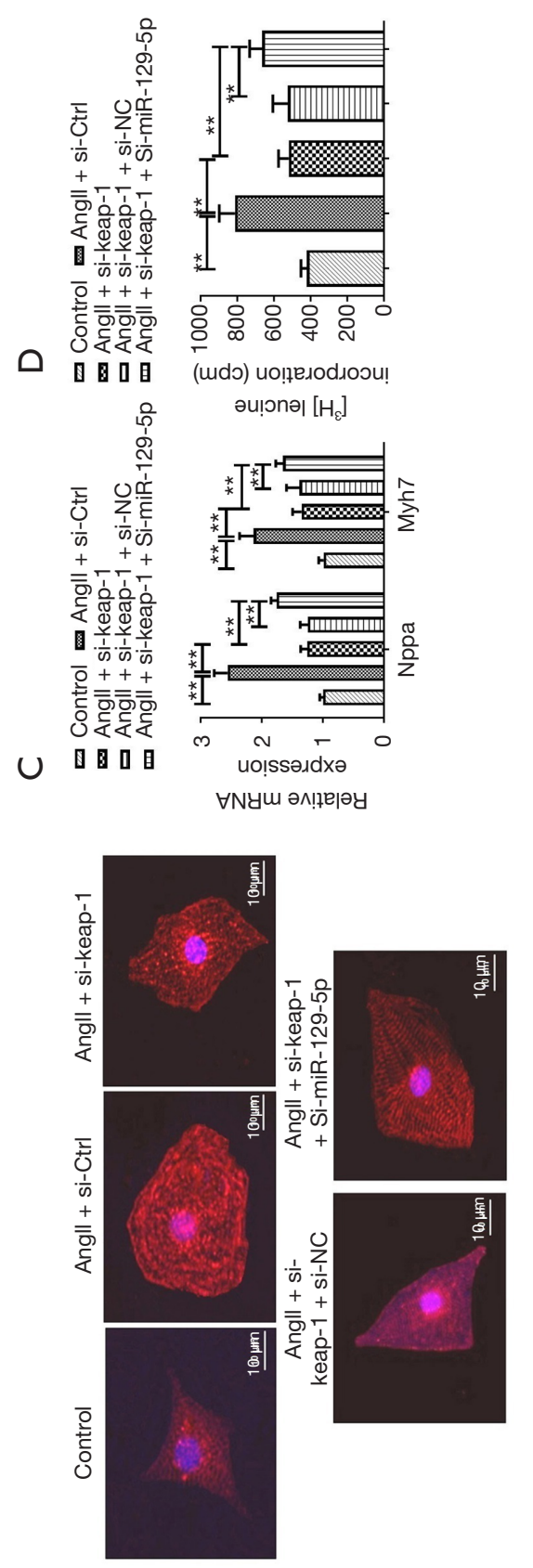

$\infty$

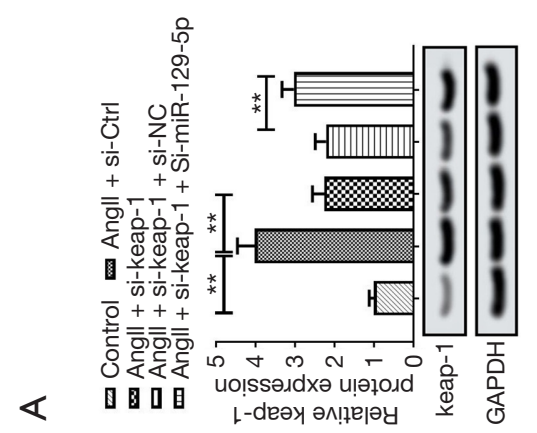

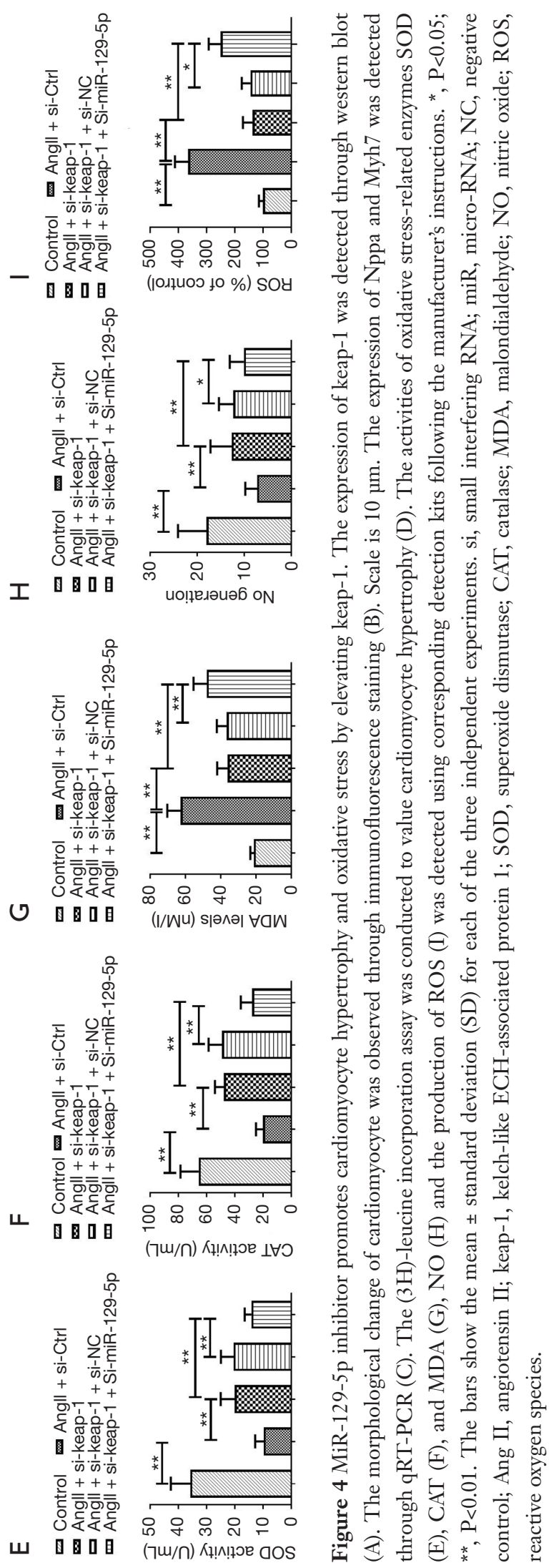

Ann Transl Med 2021;9(2):154 I http://dx.doi.org/10.21037/atm-20-8079 


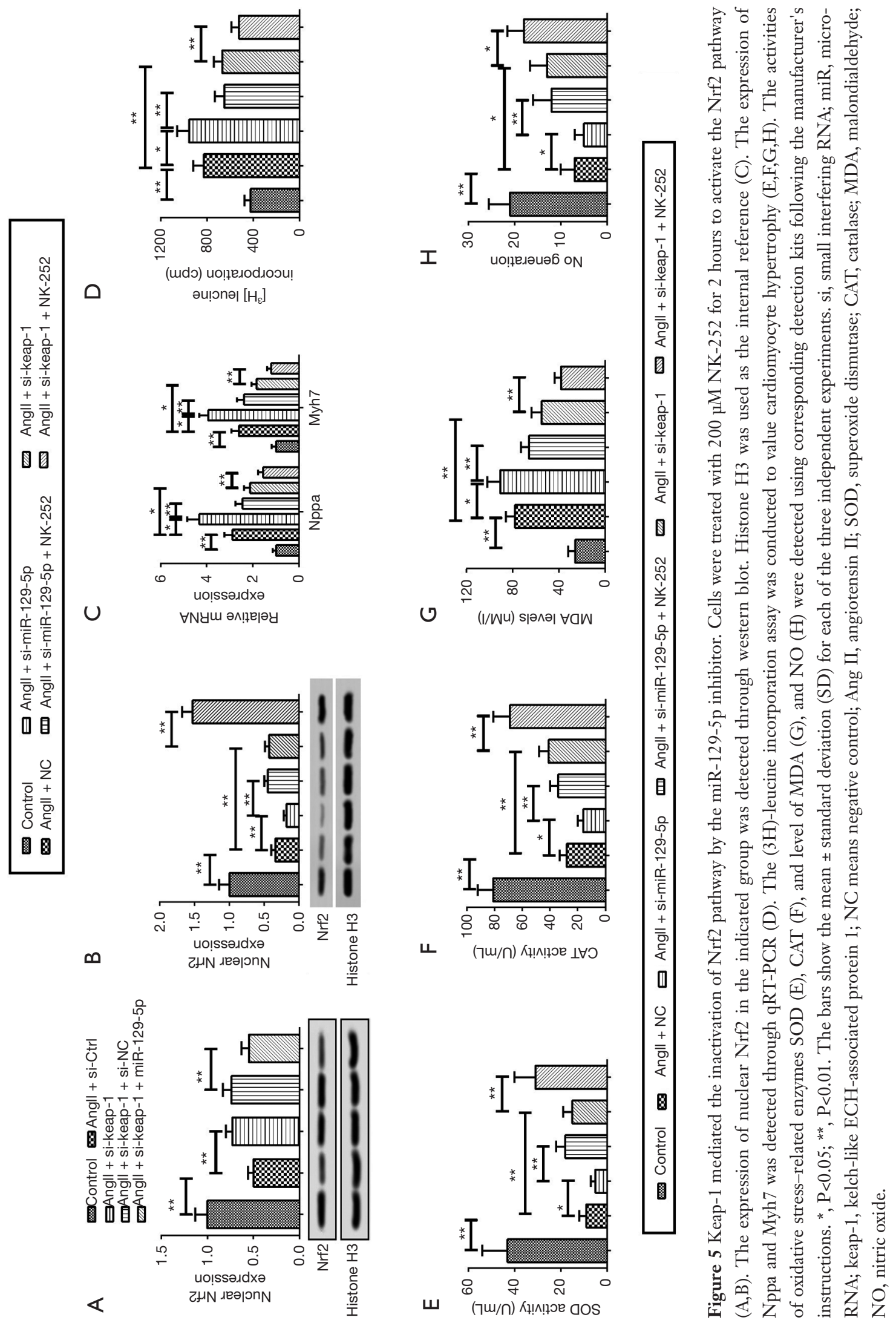


as the results could reveal the targets associated with the prevention and treatment of cardiac hypertrophy (23).

Different types of miRNAs in myocardial cells are involved in many cell biology processes, including cell proliferation, migration, and differentiation (24). Previous studies have shown that many miRNAs, such as miR-1 (25) and miR-101b (26), were down-regulated in cardiac hypertrophy, and that the overexpression of miR-1 and miR$101 \mathrm{~b}$ played an anti-hypertrophic role in the suppression of hypertrophic responses. Additionally, some other miRNAs, such as miR-297 (27) and miR-22 (28), were up-regulated in cardiac hypertrophy, and the deletion of these related miRNAs could also attenuate hypertrophic responses. Xiao et al. found that miR-129-5p was down-regulated in the serum of chronic heart failure $(\mathrm{CHF})$ patients and that the miR-129-5p mimic not only improved heart function and hemodynamic parameters, but also attenuated oxidative stress and inflammation factors in CHF rats (29). However, to date, the direct effects and the regulation mechanism of miR-129-5p in cardiac hypertrophy have not been explored. In the present study, we found that miR-129-5p was downregulated in Ang II-induced cardiac hypertrophic cells and that the overexpression of miR-129-5p successfully suppressed hypertrophic responses.

Oxidative stress is closely related to cardiac hypertrophy and is considered a major inducer for signal transduction in cardiac cells under pathological conditions (30). A large number of studies have shown that the inhibition of oxidative stress can significantly prevent and improve cardiac hypertrophy (31-33). In the present study, we found that the overexpression of miR-129-5p largely rescued the activities of the oxidative stress-related enzymes SOD and CAT. Additionally, the miR-129-5p mimic increased the generation of $\mathrm{NO}$ and cleared the accumulation of MDA, indicating that overexpressed miR-129-5p effectively suppressed Ang II-induced oxidative stress. Thus, it appears that miR-129-5p mimic may act as an antioxidant that prevents cardiac hypertrophy.

Previous studies have shown that the keap1-Nrf2 signaling pathway played an essential role in the oxidative stress defense mechanism, and that the keap1-Nrf2 signaling pathway may be a prospective target in the prevention and treatment of oxidative stress-related diseases (34,35). Keap1, the negative regulator of $\mathrm{Nrf} 2$, has an important role in the activation of keap1-Nrf2 signaling pathway (36). Notably, Liao et al. reported that miR-140-5p attenuated oxidative stress in Cisplatin induced acute kidney injury by activating the Keap1-mediated Nrf2/ARE pathway (37). In the present study, we found that the expression of keap-1 was elevated by Ang II stimulation and then suppressed by the miR129-5p mimic. At the same time, more obvious increase occurred in the protein detection than that in the mRNA detection of keap-1, which may be induced by the time and space gap between the transcription and translation of eukaryotic gene expression (38). A further study revealed a targeting relationship between miR-129-5p and keap-1. Additionally, we found that inhibiting the effect of siRNAkeap-1 on hypertrophic responses and oxidative stress was both largely neutralized in the presence of siRNA-miR129-5p.

We also explored the participatory role of the Keap1/ Nrf2 pathway in the anti-hypertrophic role of miR-129-5p. Ying et al. revealed that Phloretin prevented diabetic cardiomyopathy by the dissociating Keap1/Nrf2 complex and inhibiting oxidative stress (39). However, the specific association between Keap1/Nrf2 and cardio hypertrophy has never been clearly identified. In our study, we found that siRNA-miR-129-5p abolished the activating effect of siRNA-keap-1 on the Nrf2 pathway by strongly suppressing the nuclear transport of Nrf2. To further verify our conjecture, NK-252, a Nrf2 specific activator, was used in our study. The results showed that silenced miR-129-5p aggravated cardiomyocyte hypertrophy by promoting keap-1 mediated oxidative stress via the inactivation of the Nrf2 pathway. Thus, this is the first study to show that the activation of the Keap1/Nrf2 pathway may have therapeutic effects on cardio hypertrophy, and that there is a theoretical basis for the application of the Nrf2 pathway activator in cardiac hypertrophy.

To sum, we constructed an in vitro cardiomyocyte hypertrophy model by stimulating the HCM with Ang II. This study was the first to show that the miR-129-5p/ keap-1/Nrf2 axis could have potential therapeutic effects in cardiac hypertrophy. The graphic abstract of the present research was shown as the Figure 6. These results suggest novel research avenues for the prevention and treatment of cardiac hypertrophy. However, we acknowledge that our work had a number of limitations. For example, further research needs to be conducted to determine whether miR-129-5p overexpression could attenuate the cardiac hypertrophy in vivo model. In the future, we will conduct related research that follows on from these experiments. 


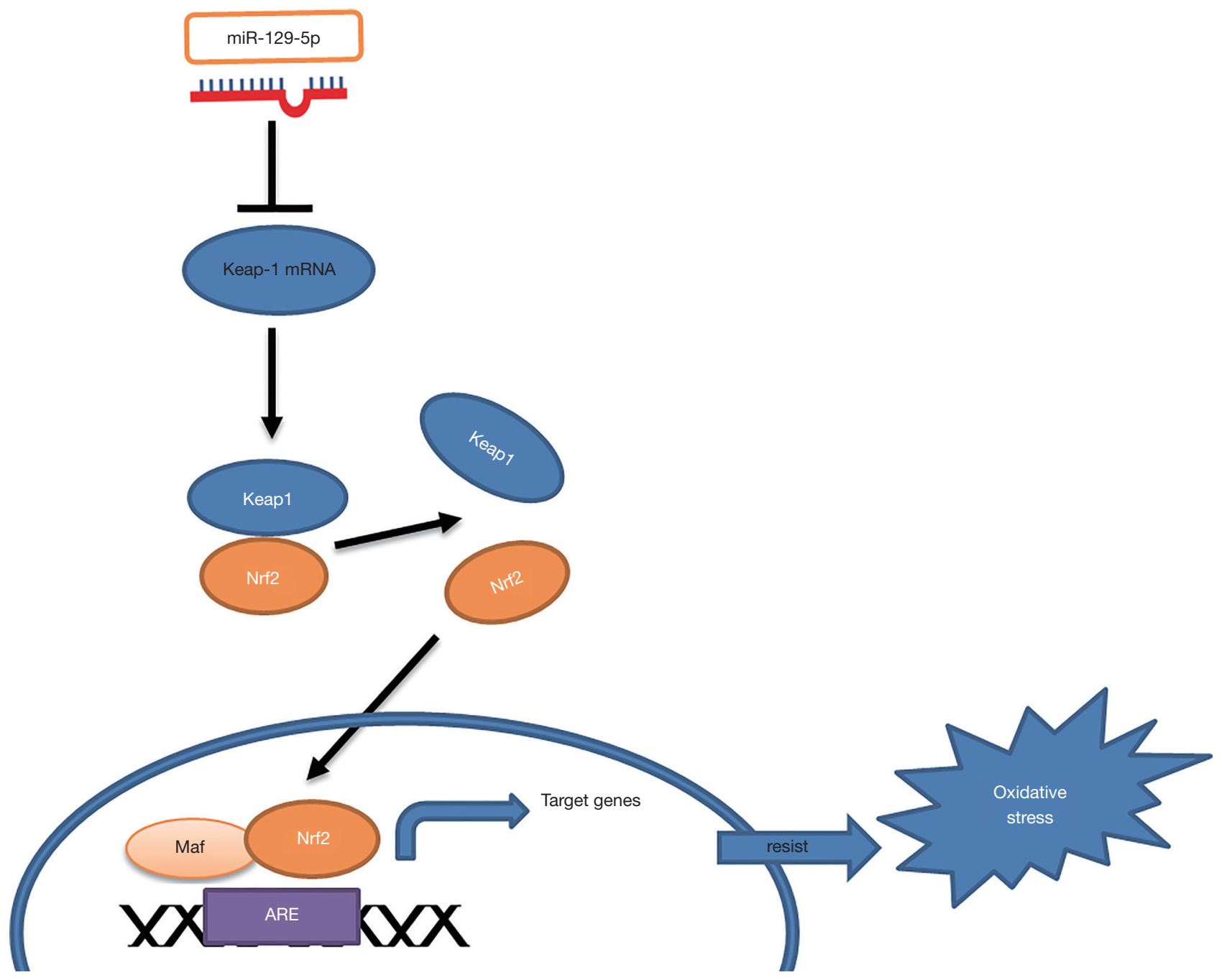

Figure 6 The Nrf2 pathway is involved in the protective effects of microRNA-129-5p in Ang II-induced cardiomyocyte hypertrophy by targeting keap-1. miR, micro-RNA; mRNA, message ribonucleic acid; keap-1, kelch-like ECH-associated protein 1; Nrf2, nuclear factor erythroid2-related factor 2; ARE, antioxidant response element.

\section{Acknowledgments}

We would like to thank the Beijing Shijitan Hospital of Capital Medical University for its assistance.

Funding: None.

\section{Footnote}

Reporting Checklist: The authors have completed the MDAR reporting checklist. Available at http://dx.doi.org/10.21037/ atm-20-8079

Data Sharing Statement: Available at http://dx.doi. org/10.21037/atm-20-8079

Conflicts of Interest: All authors have completed the ICMJE uniform disclosure form (available at http://dx.doi. org/10.21037/atm-20-8079). The authors have no conflicts of interest to declare.

Ethical Statement: The authors are accountable for all aspects of the work in ensuring that questions related to the accuracy or integrity of any part of the work are appropriately investigated and resolved. The present study was approved by the Ethics Committee of the Beijing 
Shijitan Hospital of Capital Medical University.

Open Access Statement: This is an Open Access article distributed in accordance with the Creative Commons Attribution-NonCommercial-NoDerivs 4.0 International License (CC BY-NC-ND 4.0), which permits the noncommercial replication and distribution of the article with the strict proviso that no changes or edits are made and the original work is properly cited (including links to both the formal publication through the relevant DOI and the license). See: https://creativecommons.org/licenses/by-nc-nd/4.0/.

\section{References}

1. Ferrario CM. Cardiac remodelling and RAS inhibition. Ther Adv Cardiovasc Dis 2016;10:162-71.

2. Shimizu I, Minamino T. Physiological and pathological cardiac hypertrophy. J Mol Cell Cardiol 2016;97:245-62.

3. Lifton RP, Gharavi AG, Geller DS. Molecular mechanisms of human hypertension. Cell 2001;104:545-56.

4. Piratello AC, Moraes-Silva I, Paulini J, et al. Renin angiotensin system and cardiac hypertrophy after sinoaortic denervation in rats. Clinics (Sao Paulo) 2010;65:1345-50.

5. Liu CT, Liu MY. Daily sesame oil supplementation attenuates local renin-angiotensin system via inhibiting MAPK activation and oxidative stress in cardiac hypertrophy. J Nutr Biochem 2017;42:108-16.

6. Philippidis A. Gene Therapy Briefs. Hum Gene Ther Clin Dev 2018;29:172-5.

7. Gabisonia K, Recchia FA. Gene Therapy for Heart Failure: New Perspectives. Curr Heart Fail Rep 2018;15:340-9.

8. Ylä-Herttuala S, Baker AH. Cardiovascular Gene Therapy: Past, Present, and Future. Mol Ther 2017;25:1095-106.

9. Rupaimoole R, Slack FJ. MicroRNA therapeutics: towards a new era for the management of cancer and other diseases. Nat Rev Drug Discov 2017;16:203-22.

10. Yang G, Yin B. The advance of application for microRNAs in cancer gene therapy. Biomed Pharmacother 2014;68:137-42.

11. Tatsuguchi M, Seok HY, Callis TE, et al. Expression of microRNAs is dynamically regulated during cardiomyocyte hypertrophy. J Mol Cell Cardiol 2007;42:1137-41.

12. Carè A, Catalucci D, Felicetti F, et al. MicroRNA-133 controls cardiac hypertrophy. Nat Med 2007;13:613-8.

13. Li Z, Song Y, Liu L, et al. miR-199a impairs autophagy and induces cardiac hypertrophy through mTOR activation. Cell Death Differ 2017;24:1205-13.
14. Chen QM, Maltagliati AJ. Nrf2 at the heart of oxidative stress and cardiac protection. Physiol Genomics 2018;50:77-97.

15. Chen $\mathrm{D}, \mathrm{Li} Z$, Bao $\mathrm{P}$, et al. Nrf2 deficiency aggravates Angiotensin II-induced cardiac injury by increasing hypertrophy and enhancing IL-6/STAT3-dependent inflammation. Biochim Biophys Acta Mol Basis Dis 2019;1865:1253-64.

16. Bellezza I, Giambanco I, Minelli A, et al. Nrf2-Keap1 signaling in oxidative and reductive stress. Biochim Biophys Acta Mol Cell Res 2018;1865:721-33.

17. Fukutomi T, Takagi K, Mizushima T, et al. Kinetic, thermodynamic, and structural characterizations of the association between Nrf2-DLGex degron and Keap1. Mol Cell Biol 2014;34:832-46.

18. Xiao C, Xia ML, Wang J, et al. Luteolin Attenuates Cardiac Ischemia/Reperfusion Injury in Diabetic Rats by Modulating Nrf2 Antioxidative Function. Oxid Med Cell Longev 2019;2019:2719252.

19. Fan YF, Yu ZP, Cui XY. lncRNA Colorectal Neoplasia Differentially Expressed (CRNDE) Promotes Proliferation and Inhibits Apoptosis in Non-Small Cell Lung Cancer Cells by Regulating the miR-641/CDK6 Axis. Med Sci Monit 2019;25:2745-55.

20. Yu L, She T, Li M, et al. Tetramethylpyrazine inhibits angiotensin II-induced cardiomyocyte hypertrophy and tumor necrosis factor-alpha secretion through an NF-kappaB-dependent mechanism. Int J Mol Med 2013;32:717-22.

21. Rosca MG, Tandler B, Hoppel CL. Mitochondria in cardiac hypertrophy and heart failure. J Mol Cell Cardiol 2013;55:31-41.

22. Nakamura M, Sadoshima J. Mechanisms of physiological and pathological cardiac hypertrophy. Nat Rev Cardiol 2018;15:387-407.

23. Mosterd A, Hoes AW. Clinical epidemiology of heart failure. Heart 2007;93:1137-46.

24. Wojciechowska A, Braniewska A, Kozar-Kaminska K. MicroRNA in cardiovascular biology and disease. Adv Clin Exp Med 2017;26:865-74.

25. Diniz GP, Lino CA, Moreno CR, et al. MicroRNA-1 overexpression blunts cardiomyocyte hypertrophy elicited by thyroid hormone. J Cell Physiol 2017;232:3360-8.

26. Lee JS, Yang DK, Park JH, et al. MicroRNA-101b attenuates cardiomyocyte hypertrophy by inhibiting protein kinase $\mathrm{C}$ epsilon signaling. FEBS Lett 2017;591:16-27.

27. Bao Q, Zhao M, Chen L, et al. MicroRNA-297 promotes 
cardiomyocyte hypertrophy via targeting sigma-1 receptor. Life Sci 2017;175:1-10.

28. Huang ZP, Chen J, Seok HY, et al. MicroRNA-22 regulates cardiac hypertrophy and remodeling in response to stress. Circ Res 2013;112:1234-43.

29. Xiao N, Zhang J, Chen C, et al. miR-129-5p improves cardiac function in rats with chronic heart failure through targeting HMGB1. Mamm Genome 2019;30:276-88.

30. Rababa'h AM, Guillory AN, Mustafa R, et al. Oxidative Stress and Cardiac Remodeling: An Updated Edge. Curr Cardiol Rev 2018;14:53-9.

31. Seddon M, Looi YH, Shah AM. Oxidative stress and redox signalling in cardiac hypertrophy and heart failure. Heart 2007;93:903-7.

32. Feng H, Cao J, Zhang G, et al. Kaempferol Attenuates Cardiac Hypertrophy via Regulation of ASK1/MAPK Signaling Pathway and Oxidative Stress. Planta Med 2017;83:837-45.

33. Adebiyi AO, Adebiyi OO, Owira PM. Naringin Mitigates Cardiac Hypertrophy by Reducing Oxidative Stress and Inactivating c-Jun Nuclear Kinase-1 Protein in Type I Diabetes. J Cardiovasc Pharmacol 2016;67:136-44.

34. Magesh S, Chen Y, Hu L. Small molecule modulators of

Cite this article as: $\mathrm{Ye} \mathrm{H}, \mathrm{Xu}$ G, Zhang D, Wang R. The protective effects of the miR-129-5p/keap-1/Nrf2 axis on Ang II-induced cardiomyocyte hypertrophy. Ann Transl Med 2021;9(2):154. doi: 10.21037/atm-20-8079
Keap1-Nrf2-ARE pathway as potential preventive and therapeutic agents. Med Res Rev 2012;32:687-726.

35. Deshmukh P, Unni S, Krishnappa G, et al. The Keap1Nrf2 pathway: promising therapeutic target to counteract ROS-mediated damage in cancers and neurodegenerative diseases. Biophys Rev 2017;9:41-56.

36. Suzuki T, Yamamoto M. Molecular basis of the Keap1Nrf2 system. Free Radic Biol Med 2015;88:93-100.

37. Liao W, Fu Z, Zou Y, et al. MicroRNA-140-5p attenuated oxidative stress in Cisplatin induced acute kidney injury by activating Nrf2/ARE pathway through a Keap1independent mechanism. Exp Cell Res 2017;360:292-302.

38. Tamarkin-Ben-Harush A, Schechtman E, Dikstein R. Cooccurrence of transcription and translation gene regulatory features underlies coordinated mRNA and protein synthesis. BMC Genomics 2014;15:688.

39. Ying Y, Jin J, Ye L, et al. Phloretin Prevents Diabetic Cardiomyopathy by Dissociating Keap1/Nrf2 Complex and Inhibiting Oxidative Stress. Front Endocrinol (Lausanne) 2018;9:774.

(English Language Editor: L. Huleatt) 